Supporting Information

\title{
Mechanically Robust and Reprocessable Acrylate Vitrimers with Hydrogen Bond-integrated Networks for photo-3D Printing
}

Hong Gao*, Yingchun Sun, Miaomiao Wang, Zhen Wang, Guoqiang Han, Ling Jin, Peng Lin, Youyi Xia and Kui Zhang*

School of Chemistry and Chemical Engineering, Anhui University of Technology, Ma’anshan Anhui 243032, China.

Corresponding Authors

*E-mail:

pshgao@ahut.edu.cn(Hong,Gao),

kuizhang@ahut.edu.cn (Kui Zhang) 
Table S1. The formula of GS-xAM

\begin{tabular}{cccccc}
\hline Samples codes & GS & THFA & AM & $\begin{array}{c}\text { TBD } \\
(5 \mathrm{~mol} \% \text {-COO } \\
\text { of GS })\end{array}$ & TPO \\
\hline GS-0AM & 60 & 40 & 0 & 3.6432 & 1.0364 \\
GS-5AM & 60 & 35 & 5 & 3.6432 & 1.0364 \\
GS-10AM & 60 & 30 & 10 & 3.6432 & 1.0364 \\
GS-15AM & 60 & 25 & 15 & 3.6432 & 1.0364 \\
GS-20AM & 60 & 20 & 20 & 3.6432 & 1.0364 \\
\hline
\end{tabular}<smiles>C=C(C)C(=O)OCC1OC1C(=O)OCC(O)CNC(=O)C(=O)OCC(O)C(=O)OCC(O)CC</smiles><smiles>C=C(C)C(=O)OCC(=C)C(=O)OCC(C)COC(=O)C(=C)C</smiles>

Figure S1. The reaction mechanism of acids with epoxides mediated with tetrabutylammonium bromide. 


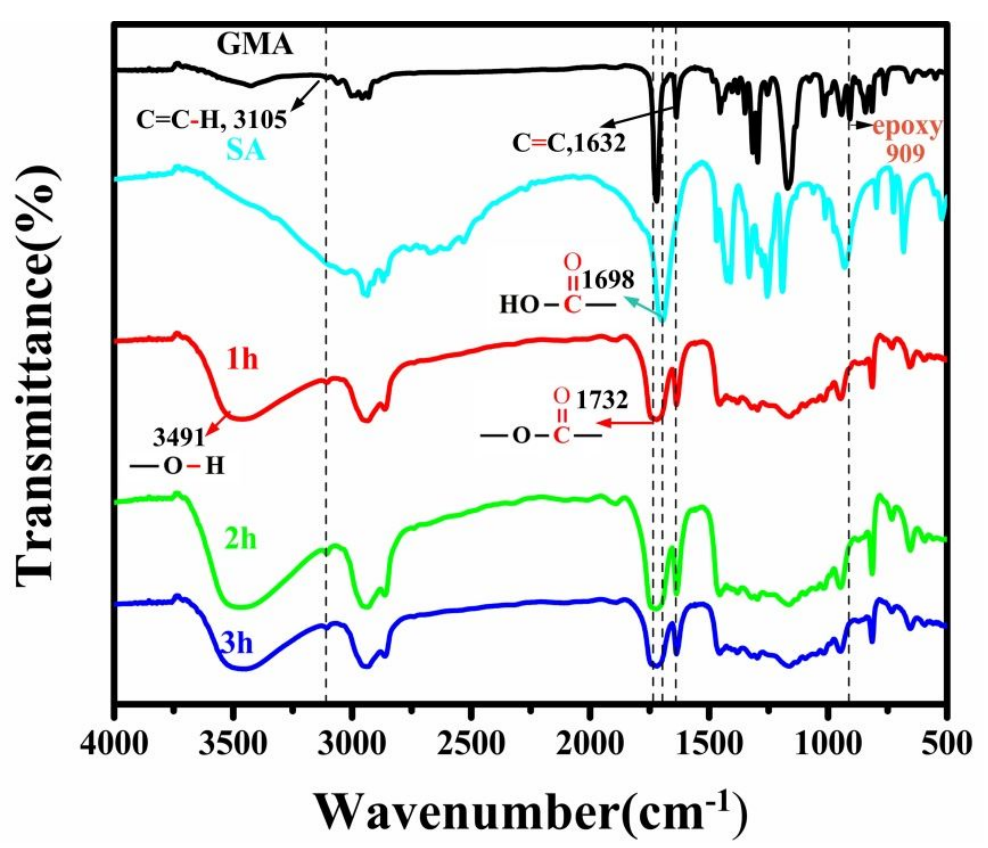

Figure S2. FTIR of GMA, SA and their reaction products at different time $1 \mathrm{~h}, 2 \mathrm{~h}$ and $3 \mathrm{~h}$.
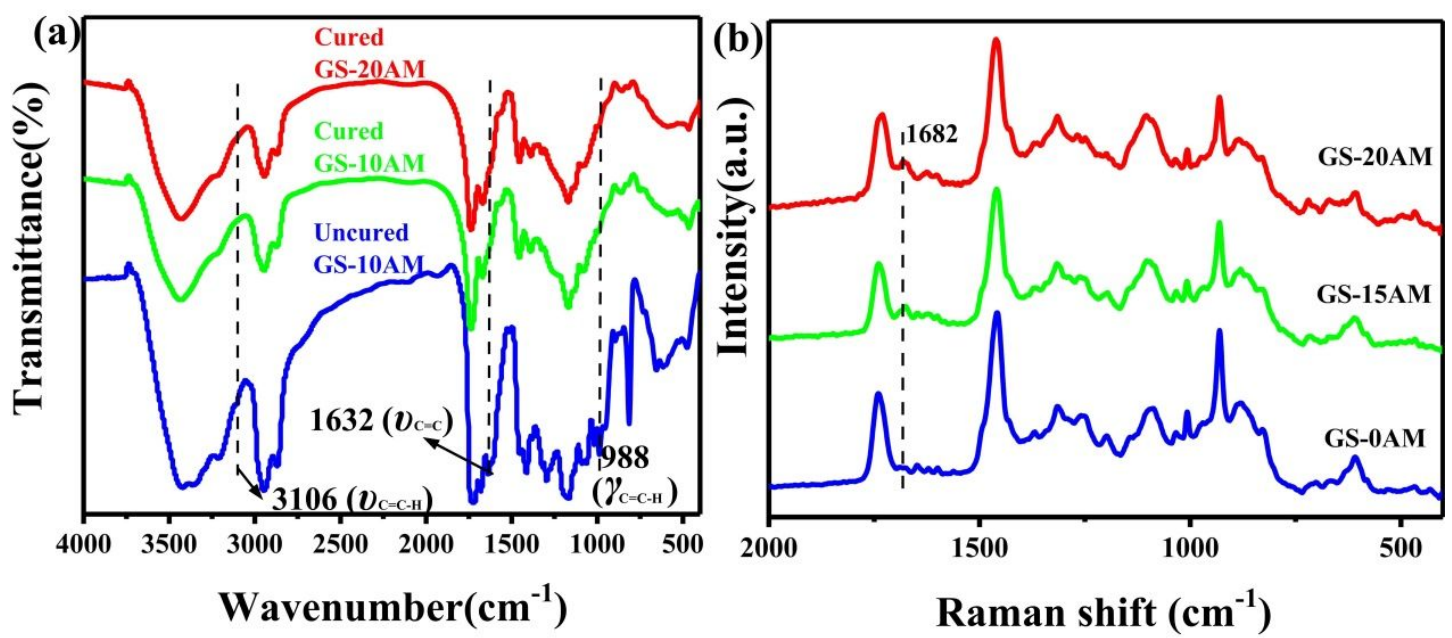

Figure S3. FTIR and Raman spectra of GS-xAM. (a)FTIR of GS-10AM before and after being cured and cured GS-20AM. (b) Raman spectra of GS-0AM, GS-15AM and GS-20AM. 


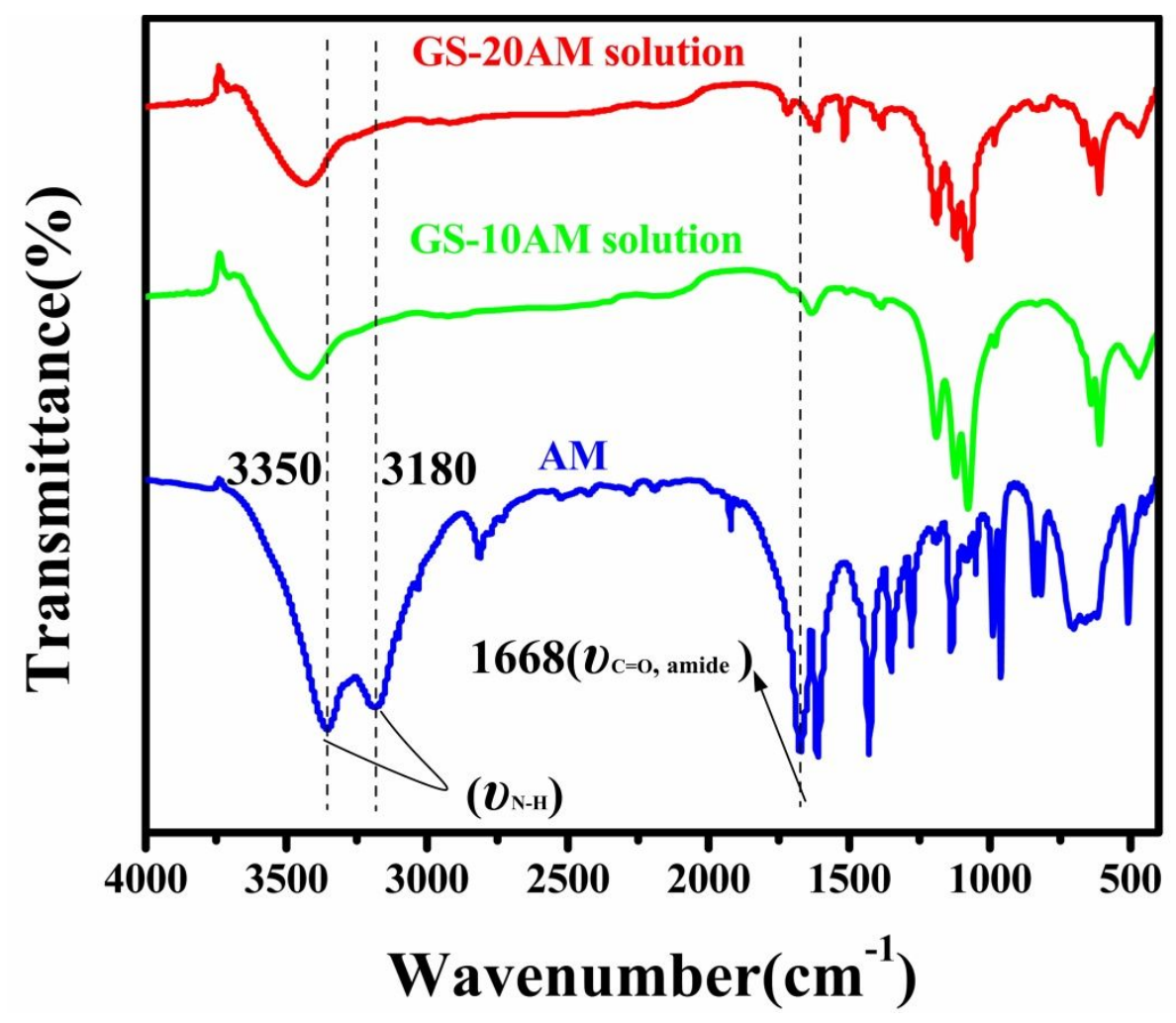

Figure S4. FTIR of acrylamide (AM) and the acetone solution immersing GS-10AM and GS-20AM.
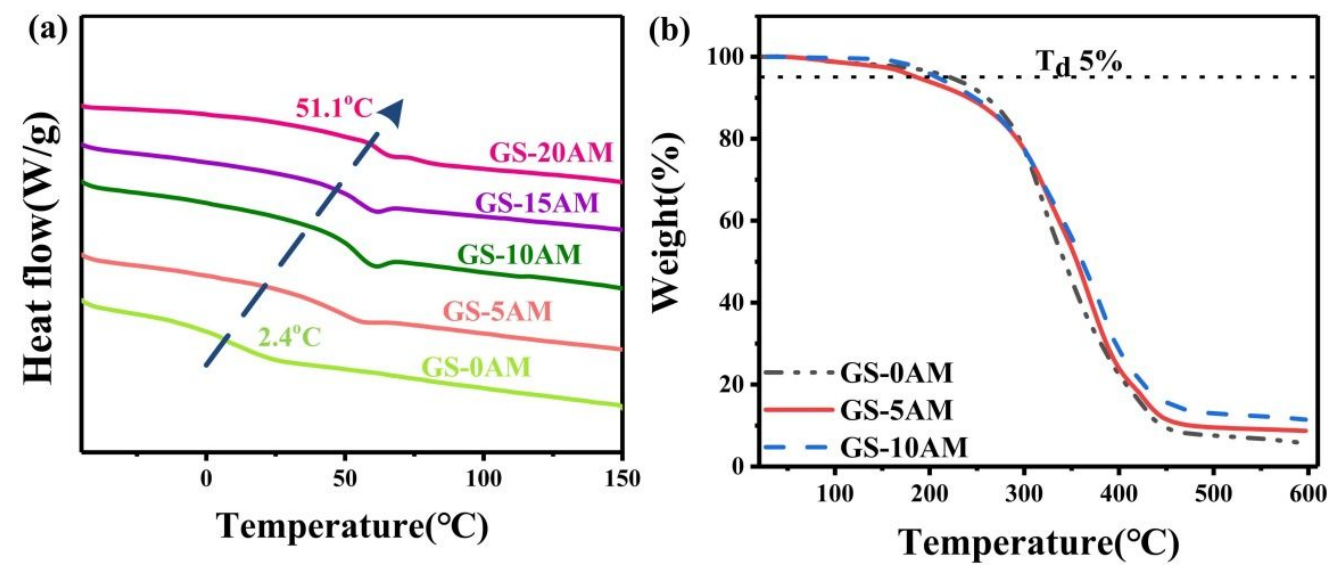

Figure S5. (a) DSC and (b) TGA curves of GS-xAM. 


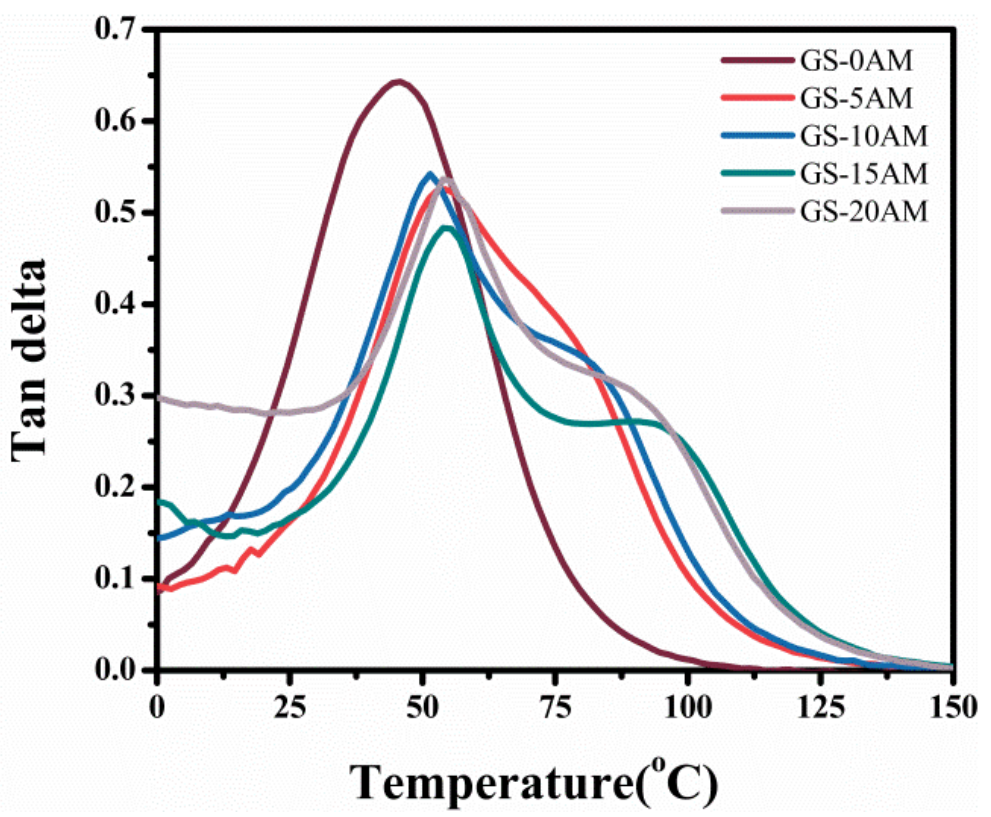

Figure S6. The tan delta curves of GS-xAM

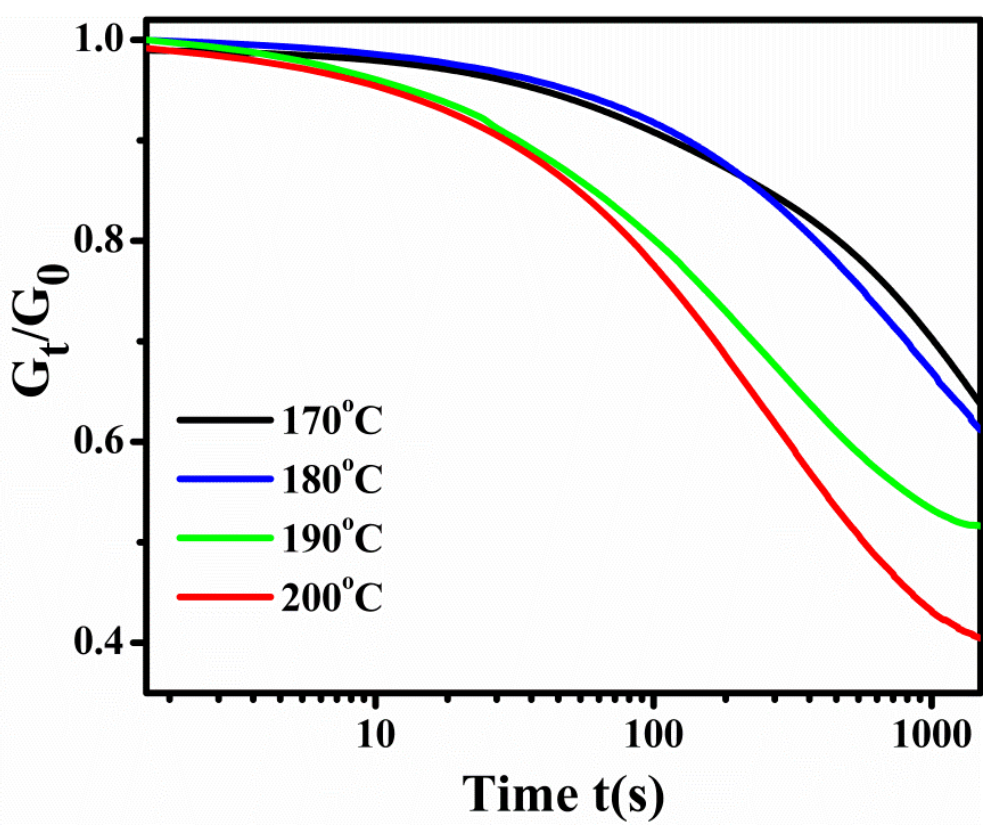

Figure S7. Normalized stress relaxation curves of GS-10AM from 170 to $200^{\circ} \mathrm{C}$. 
Table S2. The mechanical properties of GS-xAM

\begin{tabular}{cccc}
\hline Samples & $\begin{array}{c}\text { Tensile Strength } \\
(\mathrm{MPa})\end{array}$ & $\begin{array}{c}\text { Fracture Energy } \\
\left(\mathrm{MJ} / \mathrm{m}^{3}\right)\end{array}$ & $\begin{array}{c}\text { Modulus } \\
(\mathrm{MPa})\end{array}$ \\
\hline GS-0AM & $9.1 \pm 0.84$ & $1.46 \pm 0.23$ & $226 \pm 26.9$ \\
GS-5AM & $12.0 \pm 0.96$ & $1.67 \pm 0.41$ & $370 \pm 45.7$ \\
GS-10AM & $24.2 \pm 3.34$ & $2.12 \pm 0.57$ & $634 \pm 10.0$ \\
GS-15AM & $28.3 \pm 3.19$ & $2.56 \pm 0.54$ & $715 \pm 52.3$ \\
GS-20AM & $40.1 \pm 4.15$ & $2.06 \pm 0.74$ & $871 \pm 66.9$ \\
\hline
\end{tabular}

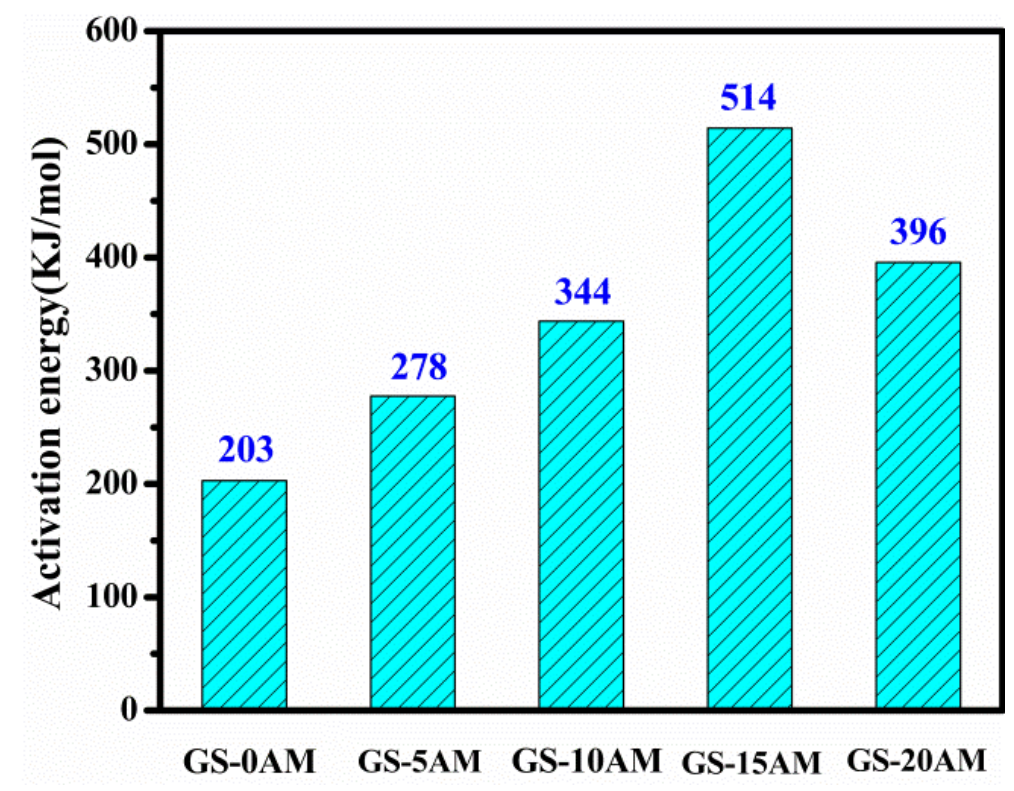

Figure S8. Activation energies of GS-xAM for the slippage of polymer chain segment, estimated from the Arrhenius plots. 


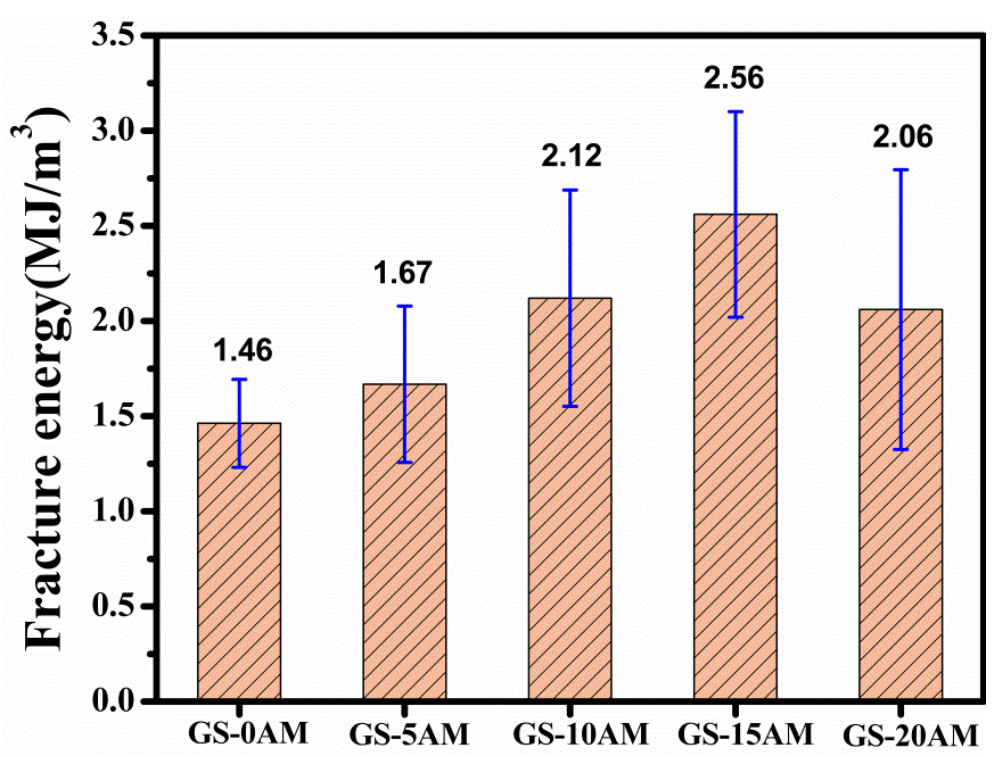

Figure S9. The fracture energy of GS-xAM.

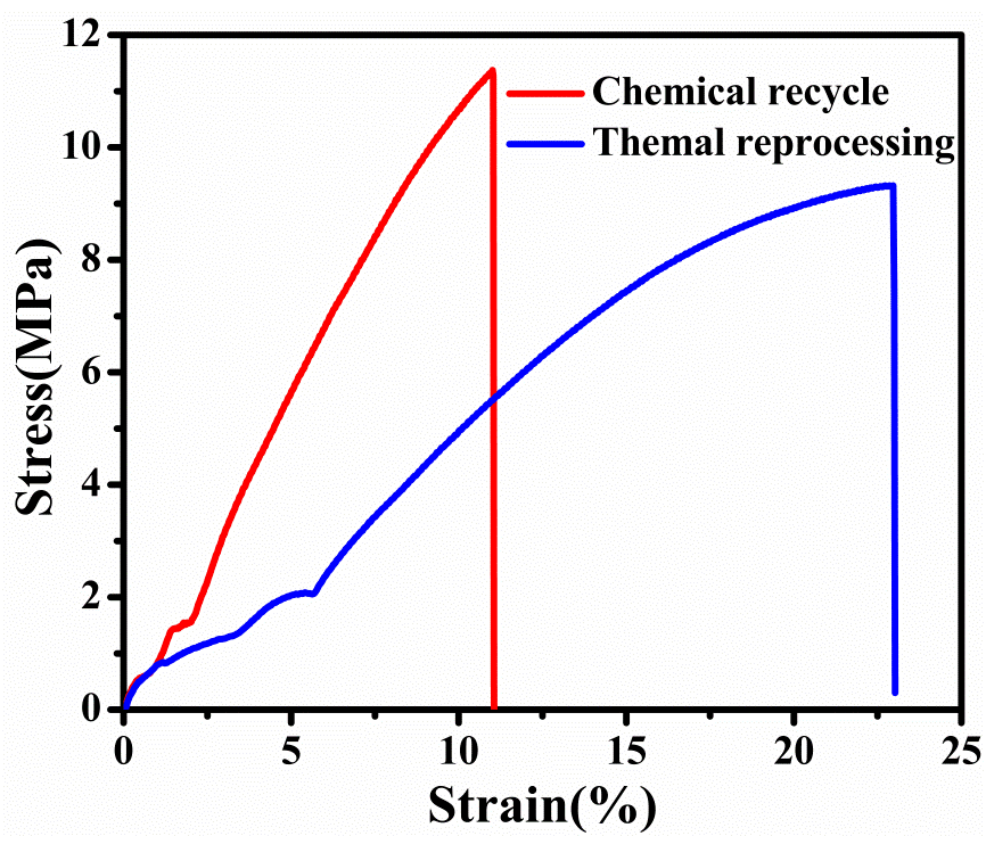

Figure S10. The stress-strain curves of GS-10AM treated after thermal reprocessing and chemical recycle. 


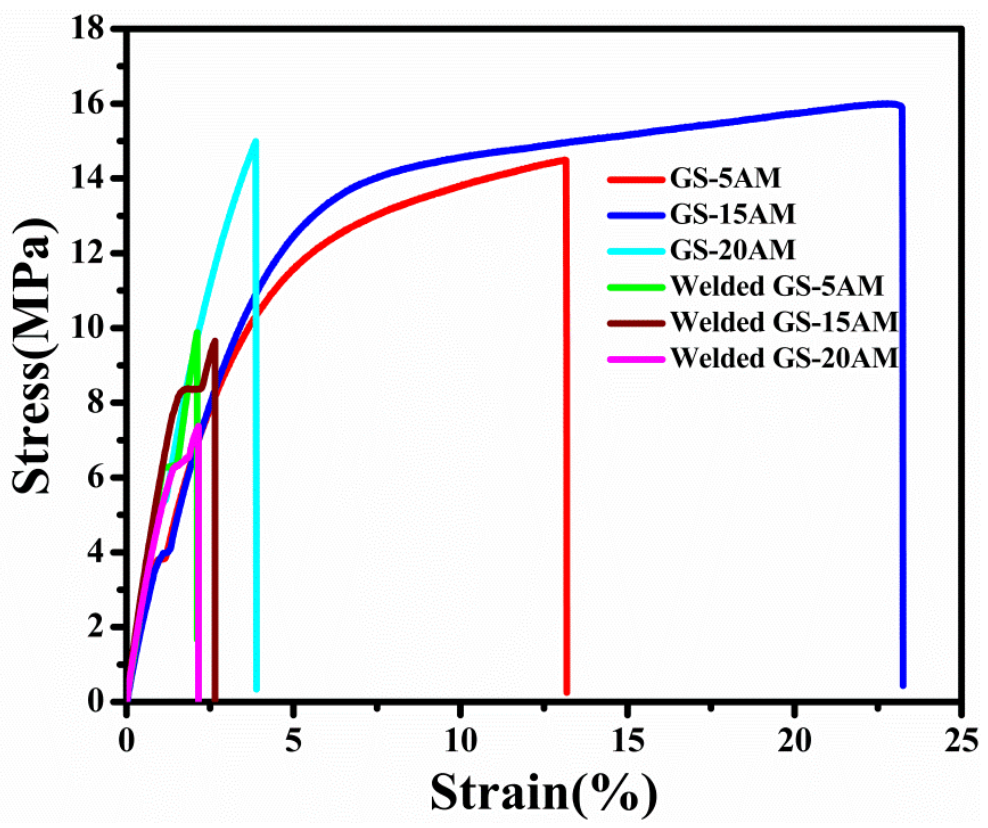

Figure S11.The stress-strain curves of lap shear tests for GS-5AM, GS-15AM and GS-20AM before and after welding. 


\section{SI Appendix}

\section{Noda's rule for the generalized 2D correlation spectra}

If the correlation intensity $\Phi\left(\mathrm{v}_{1}, \mathrm{v}_{2}\right)$ in synchronous spectra has the same symbol (positive or negative) as the correlation peak $\Psi\left(\mathrm{v}_{1}, \mathrm{v}_{2}\right)$ in asynchronous spectra, then the movement of band $\mathrm{v}_{1}$ is prior to or earlier than that of band $\mathrm{v}_{2}$, and vice versa. Besides, if the correlation intensity in synchronous spectra is not zero (or blank), but zero in asynchronous one, then the movements of bands at $\mathrm{v}_{1}$ and $\mathrm{v}_{2}$ are simultaneous.

Noda's rules are summarized as follows:

(1) If $\Phi\left(\mathrm{v}_{1}, \mathrm{v}_{2}\right)>0, \Psi\left(\mathrm{v}_{1}, \mathrm{v}_{2}\right)>0$ or $\Phi\left(\mathrm{v}_{1}, \mathrm{v}_{2}\right)<0, \Psi\left(\mathrm{v}_{1}, \mathrm{v}_{2}\right)<0$, then the movement of $\mathrm{v}_{1}$ is before than that of $v_{2}$.

If $\Phi\left(\mathrm{v}_{1}, \mathrm{v}_{2}\right)>0, \Psi\left(\mathrm{v}_{1}, \mathrm{v}_{2}\right)<0$ or $\Phi\left(\mathrm{v}_{1}, \mathrm{v}_{2}\right)<0, \Psi\left(\mathrm{v}_{1}, \mathrm{v}_{2}\right)>0$, then the movement of $\mathrm{v}_{1}$ is after than that of $v_{2}$.

${ }^{\otimes}$ If $\Phi\left(\mathrm{v}_{1}, \mathrm{v}_{2}\right)>0, \Psi\left(\mathrm{v}_{1}, \mathrm{v}_{2}\right)=0$ or $\Phi\left(\mathrm{v}_{1}, \mathrm{v}_{2}\right)<0, \Psi\left(\mathrm{v}_{1}, \mathrm{v}_{2}\right)=0$, then the movements of $\mathrm{v}_{1}$ and $\mathrm{v}_{2}$ are simultaneous.

$\Phi\left(\mathrm{v}_{1}, \mathrm{v}_{2}\right)$ and $\Psi\left(\mathrm{v}_{1}, \mathrm{v}_{2}\right)$ represent the correlation peaks in synchronous and asynchronous spectra, respectively.

\section{Time temperature superposition (TTS)}

Frequency dependent modulus of the GS-xAM samples were tested from $-50^{\circ} \mathrm{C}$ to $200^{\circ} \mathrm{Cat}$ the frequency of $1,2,5$ and $10 \mathrm{~Hz}$. Master curves of the storage modulus of GS-xAM were scaled by WLF equation. 


$$
\log \left(a_{T}\right)=\frac{-C_{1}\left(T-T_{r}\right)}{C_{2}+\left(T-T_{r}\right)}
$$

Where $a_{T}$ is the horizontal shift factor, and $T_{r}$ is the reference temperature. $C_{1}$ and $C_{2}$ are two empirical parameters, which depend on the reference temperature $T_{r}$.

\section{Master curves}

The room temperature $22^{\circ} \mathrm{C}$ is adopted as reference temperature. The storage modulus isothermal curves are drawn consisting of a data set of approximately about 100 isothermal curves. The principle of time-temperature superposition (TTS) was used to create a master curve for the sample. Each isotherm was shifted left or right along the $\log$ frequency axis relative to a reference isotherm in order to achieve maximum overlap of the data. Shifting was done so as to ensure that each curve matched the slope of its immediate neighbor, closest to the reference curve. The number of $\log$ (frequency) units through which an isotherm was translated is termed as its temperature-shift factor and is designated as $\log \mathrm{a}_{\mathrm{T}}$. By convention, shifts towards the right are designated as negative while those towards the left are positive shifts. 